\title{
SPHEROIDAL SYSTEMS
}


Garth Illingworth

Kitt Peak National Observatory

The form of the velocity and velocity dispersion profiles in elliptical galaxies, and their implications for anisotropy and mass-tolight ratio changes in ellipticals are discussed. Recent results on the luminosity dependence of the rotational properties of ellipticals are summarized, as are questions concerning the shapes of ellipticals. The relation of bulges to ellipticals, in the light of these new data, is considered.

\section{ELLIPTICALS: $V(r)$ and $\sigma(r)$ PROF ILES}

A recent paper (Davies et. al. 1983) summarized available kinematic data for some 43 elliptical galaxies. A significant fraction of these galaxies have dispersion $(\sigma)$ and velocity (V) profiles that extend out to near a de Vaucouleur's radius, $r_{e}$. I have selected a sample of these ellipticals, covering a range of luminosities, and plotted both rotation and dispersion profiles in dimensionless coordinates. These are by no means an unbiased sample of $\mathrm{V}$ and $\sigma$ profiles. They were chosen to illustrate the range of behavior of such profiles. Velocity dispersion profiles are of particular interest since we still do not know if ellipticals have massive halos, i.e., if the mass-to-light ratio, M/L, is approximately constant or increases steadily outward. Unlike disk systems in which $\mathrm{M} / \mathrm{L}$ variations can, in principle, be readily derived from the rotation curve of the gaseous component, the derivation of the run of $M / L$ with radius in ellipticals is complicated by anisotropy in the stellar velocity distribution.

$\mathrm{V}(\mathrm{r})$. The sample rotation curves have been normalized by the mean maximum rotation velocity $\left\langle\mathrm{V}_{\max }\right\rangle$, and plotted against $r / r_{e}$ in Figures 1 and 2. The galaxies in this sample have a range of ellipticities (E2E5) and are not, in general, rotationally flattened. All have $V_{\max } \geqslant 50$ $\mathrm{km} \mathrm{s} \mathrm{s}^{-1}$, so that observational uncertainties do not significantly affêct the rotation curves. Typically, $15^{\prime \prime} \leq \mathrm{r}_{\mathrm{e}} \leq 70^{\prime \prime}$ and $50 \leqslant \mathrm{v}_{\max } \leqslant 150 \mathrm{~km}$ $s^{-1}$. 


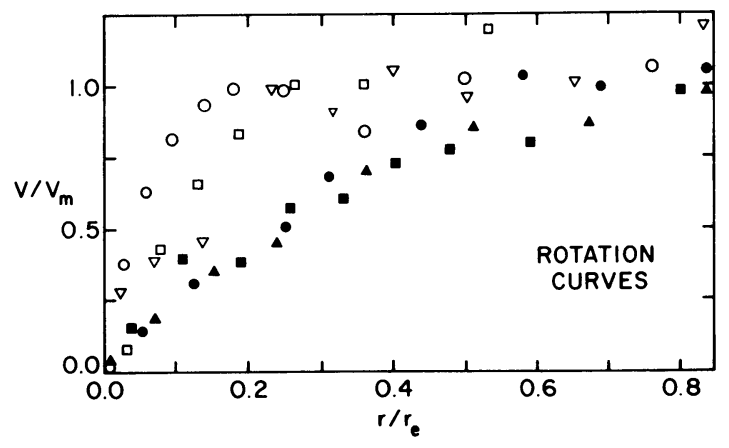

Figure 1. Absorption line rotation curves are shown for six ellipticals. The rotation velocity $V$ has been normalized by the mean maximum rotation $\mathrm{V}_{\mathrm{m}}$, and plotted against radius $\mathrm{r}$ normalized by the de Vaucouleurs radius $r_{e}$. This sample was selected to have relatively shallow gradients. Compare these with the ellipticals in Figure 2 .

Several examples of slowly rising rotation curves are shown in Figure 1. Interestingly, the three with the shallowest gradients (the filled symbols - NGC 3605, 4387, and 4478) are all low luminosity ellipticals from Davies et. al. (1983). Some ellipticals, on the other hand, show a rapid rise to $\bar{V}_{\sim} V_{\max }$ and a roughly flat rotation curve out to $r \sim r_{e}$. Examples are shown in Figure 2. The turnover radii $r_{m}$ of the three most rapidly rising rotation curves (for NGC 1052, 3377 and 4697) could be strongly affected by seeing. For example, in NGC 1052 the apparent turnover radius is $\sim 2$ ", making the actual turnover radius $\leq 300 \mathrm{pc}$. It will be interesting to see if the ellipticity remains large at such small radii as well, suggesting that anisotropy is significant even close to the nucleus (see Jedrzejewski et al. 1983, Davies and Illingworth 1983c).

While the sample is small most normal ellipticals show no significant rotation along the projected figure minor axis. To date it is only those ellipticals whose apparent principal axes show gross position angle changes (e.g., NGC 596 - Schechter and Gunn 1979, and Williams 1981), or those showing obvious signs of a recent interaction (e.g. NGC 4125, Bertola 1981, 1983), that show clear differences between the position angles of the kinematic and photometric major axes. More minor axis rotation data and dispersion profiles would be valuable.

$\sigma(r)$. Velocity dispersion profiles are shown in Figures 3 and 4, normalized to the central dispersion $\sigma_{0}$ and to $r_{e}$ as before, but plotted on logarithmic scales. Again the galaxies plotted cover a range of ellipticities and luminosities. Error bars have not been plotted in the interest of clarity; the point-to-point scatter for each galaxy gives some indication of the uncertainty involved. Most elliptical galaxies show $\sigma(r)$ decreasing with radius, as exemplified in Figure 3. This is the behavior expected for a constant M/L galaxy (see e.g., model fits for NGC 3379 in Davies and Illingworth 1983a) with an isotropic velocity 


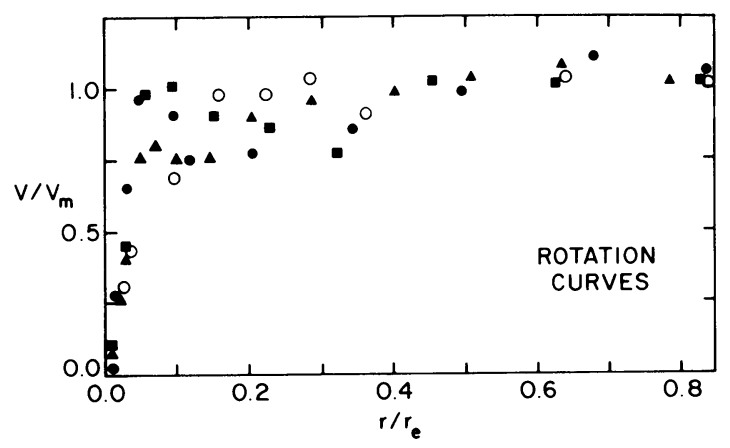

Figure 2. As for Figure 1, but for a sample of ellipticals with rapidly rising rotation curves that are roughly flat beyond the turnover.

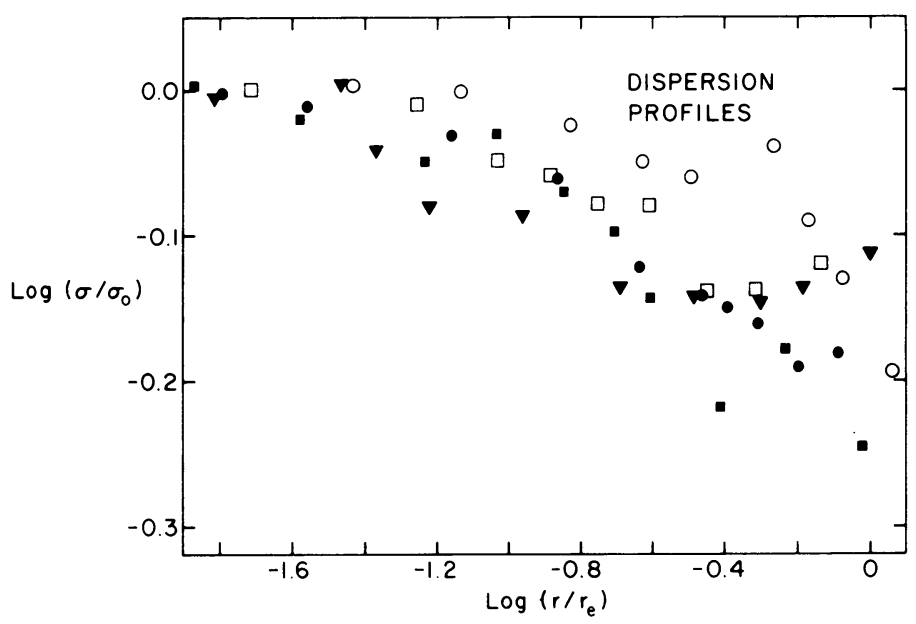

Figure 3. Velocity dispersion profiles for a sample of five ellipticals, normalized by the central dispersion $\sigma_{0}$, and by $r_{e}$ as in Figure 1, and plotted on logarithmic scales. This sample was selected to have decreasing $\sigma(r)$ profiles.

dispersion tensor. Alternatively, if $\mathrm{M} / \mathrm{L}$ increases outwards as doctrine would have it, then the radial component $\sigma_{r}$ of the velocity dispersion must become increasingly important at the expense of the azimuthal component $\sigma_{t}$ such that the observed (line-of-sight projection) dispersion decreases. In terms of the usual anisotropy parameter, $\beta=1-\left(\sigma_{t} / \sigma_{r}\right)^{2}, \beta \rightarrow 1$ as the radius increases $(\beta=0$ for isotropy and $\beta \rightarrow-\alpha$ in azimuthally anisotropic systems). Such radial anisotropy is not unreasonable given that collapse models of galaxy formation are likely to result in velocity distributions with radially elongated velocity ellipsoids.

While the majority of ellipticals show $\sigma(r)$ profiles that decrease 
with radius, a significant fraction have $\sigma(r)$ constant or even increasing. Several examples of constant $\sigma(r)$ profiles are shown in Figure 4. The $C D$ galaxy in the center of the cluster A2029 has a $\sigma(r)$ profile that increases significantly with radius (Dressler 1979). The comparison of his observed dispersion profile with that expected for a constant M/L model is striking (his Figure 5); at $\mathrm{r} \sim 100 \mathrm{kpc}$,

$\sigma_{\text {obs }} \sim 2 \sigma_{\text {model }} \cdot$

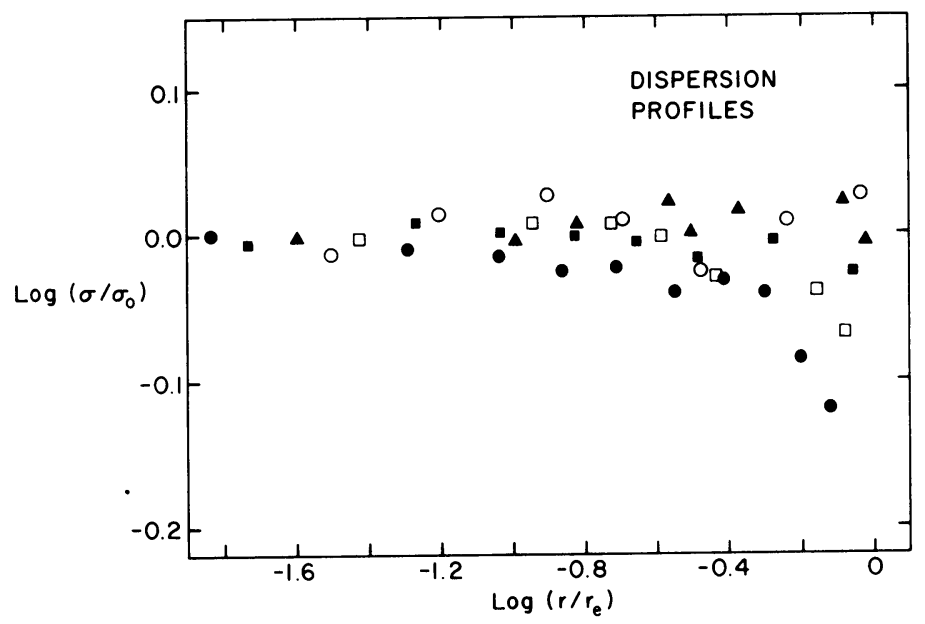

Figure 4. As in Figure 3, but for a sample of ellipticals whose dispersion profiles are approximately constant with radius. An interesting feature of this $\log -\log$ plot is that one galaxy is seen to have a very rapid change in slope (filled circles). This is unlikely to be real and probably indicates a problem with sky subtraction at the very low surface brightness levels involved. On the usual linear $\sigma(r)$ plots this problem was not as apparent.

These flat or slowly rising dispersion profiles are inconsistent with constant $\mathrm{M} / \mathrm{L}$, isotropic-dispersion models. However, as before, anisotropy changes can mimic $M / L$ changes. In these cases, the azimuthal component of the dispersion would need to increase at the expense of the

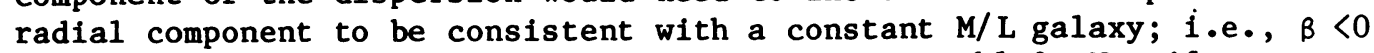
(see e.g., Tonry 1983). Is this physically reasonable? Not if ellipticals form by the usual dissipational or dissipationless collapse picture. It is not at all apparent how such a galaxy could come to have an azimuthally elongated velocity ellipsoid. However, if mergers of disk galaxies contribute significantly to the elliptical population, such anisotropy may be quite reasonable, as Tonry (1983) has argued. There is no theoretical support, though, for this situation. It would be valuable if $\mathrm{N}$-body models of disk-system mergers that result in elliptical-like objects with appropriate values of $\sigma$ and $\mathrm{V} / \sigma$, could be investigated to see if $\sigma_{t}>\sigma_{r}$ in the body of the model. 
In summary, it appears possible for ellipticals to be constant $\mathrm{M} / \mathrm{L}$ objects. We would require some galaxies to have radially-anisotropic envelopes and some to have azimuthal anisotropy. Whether such a situation stays tenable as individual galaxies are investigated in more detail remains to be answered. For it is clear from the models of flat $\sigma(r)$ profile galaxies (Efstathiou 1982, Tonry 1983) that anisotropy can only compensate for the decrease in $\sigma_{\text {total }}$ out to $r \sim r_{e}$; beyond that radius $\sigma_{\text {obs }}$ decreases because essentially all the dispersion is in the azimuthal component. Measurement of $\sigma$ for $r>r_{e}$ in ellipticals with increasing $\sigma(r)$ would be valuable. Tonry's (1983) fit to Dressler's (1979) A2029 data (his Figure 4) exemplifies the problem. With $\mathrm{r}_{\mathrm{e}}-100$ $\mathrm{kpc}$, Tonry managed to fit the dispersion profile with a constant $\mathrm{M} / \mathrm{L}$ but highly anisotropic model (with $\beta \sim-15$ ). However, $\mathrm{r}_{\mathrm{e}}<100 \mathrm{kpc}$ in A2029, being probably more like $70 \mathrm{kpc}$. In this case, it is likely that the $\mathrm{M} / \mathrm{L}$ will need to increase with radius, regardless of the amount of anisotropy present. Of course, the actual change in M/L will depend greatly upon the degree of anistropy, being greatest in the isotropic models suggested by Dressler and least in a low $\beta(\beta<<0)$ model.

A sufficiently large sample (e.g., Davies et. al. 1983) of elliptical galaxy dispersion profiles now exist that we can investigate the distribution of $\sigma$ trends with radius (Fried and Illingworth $1983 \mathrm{~b})$. For example, by fitting power laws to $\sigma(r)$ we find that $d(1 \mathrm{n}$ o) $/ d($ in $r)$ ranges from 0.1 to -0.3 , i.e., slightly increasing as in A2029 to decreasing as in NGC 3379. The median value lies between 0.0 and -0.1 , larger than the $\sim-0.15$ typical of constant M/L isotropic models. There appear to be no significant trends with ellipticity and luminosity. This, as well as the detailed $\sigma(r)$ and $V(r)$ profiles, will be discussed more extensively in Fried and Illingworth (1983b).

\section{LM INOS ITY DEPENDENCE OF ROTATION PROPERT IES}

The usual $V / \bar{\sigma}$ vs $\varepsilon$ plot is shown in Figure 5 , where $V$ is the maximum rotation velocity, $\sigma$ is the mean velocity dispersion within $\mathrm{r}_{\mathrm{e}} / 2\left(\bar{\sigma} \sim \sigma_{0}\right.$, the central dispersion; see Davies et. al. 1983), and $\varepsilon$ is the ellipticity. The oblate line defines where rotationally-flattened oblate galaxies fall irrespective of inclination, whereas the prolate line shows the median of the distribution expected for rotationallyflattened prolate galaxies (Binney 1978, nlingworth 1981). The filled symbols are for ellipticals brighter than $M_{B}=-20.5$. Note that there are very few oblate rotators ( $\leq 15-20 \%)$, and that these bright ellipticals are not consistent with being prolate rotators.

The open symbols are for ellipticals fainter than $M_{B}=-20.5$ (and one likely SO at $\mathrm{V} / \sigma>1.0$ and $\varepsilon=0.33$ ). All these galaxies are consistent with being oblate rotators. This striking result is taken from Davies et. al. (1983). This variation of rotational properties is better demonstrated in Figure 6 . Here the $\varepsilon$ dependence of $V / \sigma$ has been removed by normalizing each observed $V / \sigma$ by that expected for an oblate rotator at the appropriate $\varepsilon$. This parameter, 


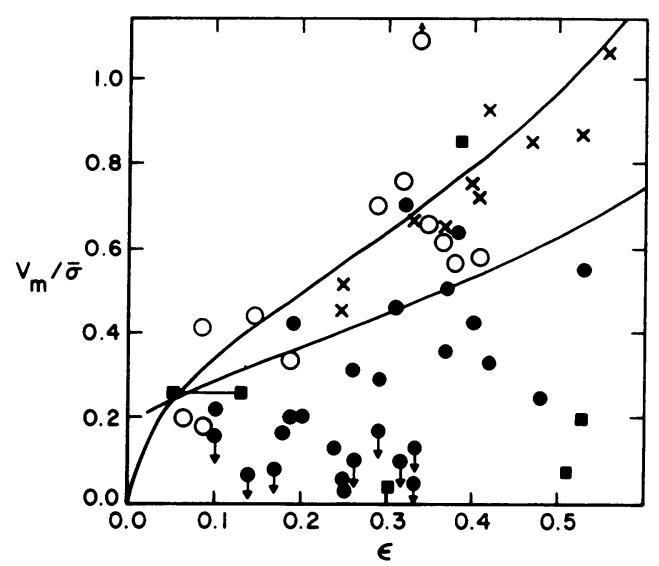

Figure 5. The maximum rotation velocity $\mathrm{V}_{\mathrm{m}}$, normalized by the mean dispersion $\bar{\sigma}$ within $r_{e} / 2$ is plotted against ellipticity $\varepsilon$ for 32 ellipticals (filled symbols) brighter than $M_{B}=-20.5$, and for 11 ellipticals (open circles) fainter than $M_{B}=-20.5$ (and one probable $\mathrm{S} 0$ with $\left.V_{m} / \bar{\sigma}>1\right)$. Like data for the bulges of disk galaxies are indicated by crosses (see $\S \mathrm{IV}$ ). The oblate (upper) and prolate (lower) model lines are explained in the text. Arrows indicate upper limits. The two filled connected squares indicate the range of $\varepsilon$ seen in NGC 3379 for $r \lesssim r_{e}$ (Davies and Illingworth 1983a).

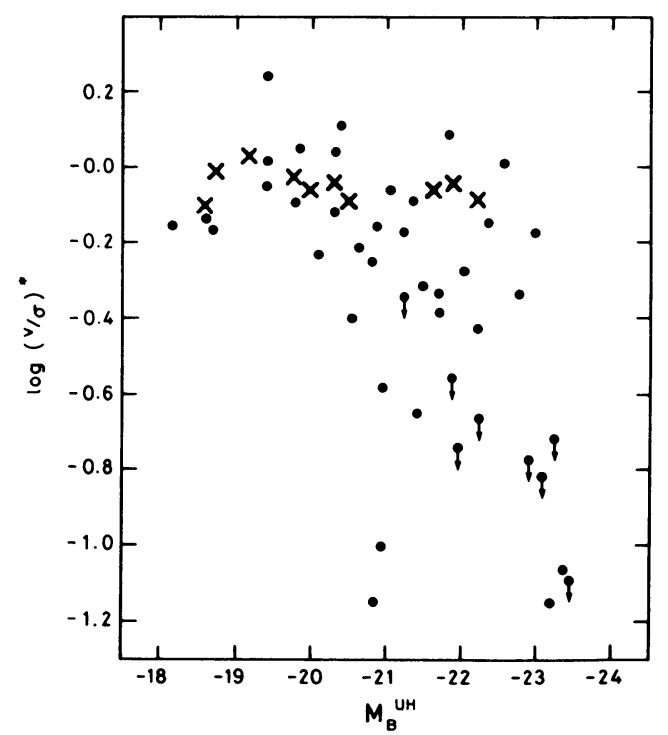

Figure 6. The observed $V_{m} / \bar{\sigma}$ for each elliptical in Figure 5 has been normalized by $\mathrm{V} / \sigma$ from the oblate line in that figure at the appropriate ellipticity, and plotted, for all $\mathrm{M}_{B}$, as filled symbols against absolute magnitude derived assuming a uniform Hubble flow model with a Hubble constant $\mathrm{H}_{0}=50 \mathrm{~km} \mathrm{~s} \mathrm{Mpc}^{-1}$. Use of Virgocentric flow magnitudes makes no significant difference to the distribution. The crosses are bulges (see $\S \mathrm{IV}$ ). 
$(\mathrm{V} / \sigma) *=(\mathrm{V} / \sigma)_{\mathrm{obs}} /(\mathrm{V} / \sigma)_{\text {Model }}=1$ for oblate rotators. Log $(\mathrm{V} / \sigma) *$ is plotted against absolute magnitude $M_{B}$ for all the ellipticals of Figure 5. In the mean, a trend is seen in that fainter ellipticals rotate more rapidly, in a dimensionless sense. However, it must be remembered that the scatter for $M_{B}<-21$ is real, and that the data tell us that slowlyrotating low-luminosity ellipticals are rare. Any formation theory for elliptical galaxies must reproduce the distribution in Figure 6. A first attempt (see Davies et. al. 1983) shows that this constraint is a severe one, particularly for hierarchical clustering collapse models, both with and without dissipation.

\section{SHAPES OF ELLIPTICALS}

A variety of statistical tests aimed at establishing the intrinsic shapes of ellipticals have been applied to photometric and kinematic data of these galaxies (e.g., Marchant and 01sen 1979, Richstone 1979, Lake 1979, Merritt 1982). Generally, such tests have shown that ellipticals are more likely to be oblate, but with very marginal significance. In addition, a potentially serious problem arises with these tests if there exist correlations between the physical properties of galaxies (e.g., M/L) and their intrinsic flattening, as has been suggested by Terlevich et al. (1981). They found, for example, that the velocity dispersion at a given absolute magnitude appears to be correlated with flattening. More and better data, both photometric and kinematic, are needed before these tests can place statistically significant constraints on the shapes of ellipticals.

For the moment only the distribution and kinematics of gas and dust in ellipticals (see e.g., Bertola and Galletta 1978, Tohline et al. 1982), and the $V(r)$ and $\sigma(r)$ data (see e.g., Davies et. al. 1983) can be used to constrain the shapes of ellipticals. From these latter data we know that ellipticals are not rotationally-flattened isotropicdispersion prolate objects. For the more luminous ellipticals, at least, little more can be established from their distribution in Figure 5. Their figures could range from prolate through triaxial to oblate, flattened, in all cases, as a result of an anisotropic velocity dispersion tensor. However, we have seen that low luminosity ellipticals are consistent with being oblate rotators. Since the photometric and kinematic properties of ellipticals appear to change smoothly with luminosity, one could argue that there is unlikely to be a dichotomy in the shapes of ellipticals, and that the more luminous galaxies are also oblate, or if triaxial are closer to being oblate than prolate. This argument is not very compelling, being based mainly on Occam's Razor and so should not be given too much weight. Clearly, observational programs on ellipticals should include galaxies with a range of luminosities and should compare, in particular, the properties of those brighter than $M_{B}=-20.5$ with those of fainter galaxies.

E11ipticals with gas and dust may provide clues as to their structure. Bertola and Galletta (1978) claimed that galaxies with gas 
and dust along their minor axes are prolate objects, initiating a lively and ongoing controversy. These early-type galaxies, generically known as polar ring galaxies, are characterised by an extensive ring of gas, dust, and sometimes an obvious young stellar population, whose rotation axis appears to be orthogonal, or nearly so, to that of the central galaxy. Examples are NGC 2685, the "Spindle", and Centaurus A, NGC 5128 .

While it is clear that stable orbits about the long axis do exist in prolata with slow or zero figure rotation, such orbits are not unique to prolate figures. Stable polar orbits do exist in triaxial forms, and in very mildly triaxial forms at that. A recent paper by SteimanCameron and Durisen (1983) shows that for a nearly oblate figure flattened by $2: 1$, i.e., an E5 galaxy, in which the axes in the plane differ by only $2 \%$ (i.e., $a: b: c=1.0: 0.98: 0.5$ ), fully $15 \%$ of any infalling material will ultimately reside along the minor axis. That is, gas will dissipate onto the short axis plane from a very significant fraction (15\%) of configuration space for the orbital angular momentum vector of the infalling material, even though the triaxiality in this case is very small. Thus, these 'prolate' figures could in reality be nearly oblate figures. Those in which the infalling material ultimately dissipates onto the long axis plane become merely 'normal' SO and $\mathrm{Sa}-\mathrm{Sb}$ galaxies.

We cannot establish, of course, that any individual galaxy is a mildly triaxial, nearly oblate figure. However, we can check the prolate hypothesis for consistency with the data. There are already indications that two objects of this class, NGC 2685 (Schechter and Gunn 1978) and NGC 4650A (Ulrich and Schechter 1983), are not prolate. Both galaxies show large rotation velocities in a dimensionless $\mathrm{V} / \sigma$ sense, and, in one case, there are indications that the rotation curve turns over and that the object rotates differentially. Such rapidly rotating objects, especially if they are rotating differentially, appear to be quite difficult to construct as stationary prolata. Yet even a quite slow figure rotation moves the stable orbits away from being polar, and ultimately, as the figure rotation increases, no stable polar or near polar orbits exist. Thus, while these polar ring or minor axis gas and dust-lane galaxies do not provide unambiguous evidence for a particular shape, they are still a valuable shape diagnostic and further study will be interesting (see e.g., Fried and Illingworth 1983a, Illingworth and Davies 1983).

In summary, no data exist that uniquely require any ellipticals to be prolate or nearly so. Assuming ellipticals to be oblate, or oblatetriaxial is consistent with available data.

\section{BULGES OF DISK GALAX IES}

Illingworth and Schechter (1982) and Kormendy and Illingworth (1982) obtained velocity and velocity dispersion profiles for 10 bulges 
of both S0 galaxies and later-type spirals. All are consistent with being rotationally-flattened oblate systems (see Figure 5), particularly when the flattening due to the disk potential is taken into account. Dressler and Sandage (1983) have further increased the sample of bulges with a similar result. The dynamical difference between bulges and ellipticals found by the above authors is now seen to be primarily a luminosity effect. As the above authors had noted, rapidly rotating bulges with absolute magnitudes $M_{B} \geqslant-20$ were being compared to slowly rotating ellipticals with $-23<M_{B}<-21$. The low luminosity ellipticals $\left(M_{B}>-20\right)$ studied recently by Davies et. al. (1983) show dynamical properties similar to bulges (Figure 5 and $\$$ II). Are bulges to be viewed once again as little ellipticals with a disk? Probably, but with some interesting differences in detail, as we shall see.

Before discussing these differences, I want to emphasize again an observational problem that can seriously bias velocity and velocity dispersion data in mixed population systems, e.g., measuring bulge properties where there is some disk contamination. Whitmore (1980) showed that velocity dispersion measurements in two component averages give higher weight to the narrow line component (i.e., the low dispersion component). Illingworth and Schechter (1982) confirmed this and found an even stronger effect for velocity measurements (see also McElroy 1983). They found that velocity measurements in two component averages typically weighted the narrow line component by a factor 3 or more, whenever it contributed $\geqslant 10 \%$ to the average. However, the weight factor depends upon the relative contribution of the two components, their velocity dispersions, and also their velocity difference. Since as little as $10 \%$ of the narrow line component can significantly affect the measurement, one should be very careful, for example, when determining bulge rotations in the presence of disk components. If disks are typically 21-22 mag arcsecond 2 in the bulge, then errors can be introduced even at bulge surface brightnesses of 19 mag arcsecond ${ }^{-2}$, i.e., one will derive dispersions that are too low and rotation velocities that are too high. While such problems have been found to occur with the Fourier quotient method, similar effects will probably be found with the Tonry-Davis (1980) CCF method and others.

For velocity measurements, at least, it should be possible to test for the effect of the narrow line component by restricting the frequency range of the fit in the Fourier plane, or equivalently by convolving the data by an appropriate (e.g., Gaussian) broadening function. Likewise for velocity dispersions, through considerable care will be required since one is attempting to derive line widths in this case.

As we mentioned above, are we now to assume that bulges are but little ellipticals with a disk, or are there still significant differences? While more data are really needed to answer this question it does appear that some interesting differences still exist. First we can try and establish the luminosity dependence of the rotational properties of bulges, i.e., how does $\mathrm{V} / \sigma$ vary with $\mathrm{M}_{\mathrm{B}}$. Is rotation dynamically less important for more luminous bulges, and do they show 
the scatter in $(\mathrm{V} / \sigma) *$ seen for bright ellipticals? The answer is a tentative no. Tentative because luminous bulges are rare. Kormendy and Illingworth's (1982) bulge sample contained only one, NGC 4594, the Sombrero. Davies and Illingworth (1983a) discuss a further two examples. Al1 three have $-22.5<\mathrm{M}_{\mathrm{B}}<21.5$, and all show significant rotation. This can be seen in Figure 8 where the bulges (crosses), unlike the ellipticals, appear to show no trend with luminosity. However, this is a small sample. Dynamical data for more luminous bulges would be valuable (see also Dressler and Sandage 1983).

There are a further three areas where bulges may be compared with ellipticals, but where the data are lacking or ambiguous, and so further observations are needed. First, as mentioned above, luminous bulges are rare, suggesting that the luminosity function for bulges differs from that for ellipticals. This needs to be quantified. Second, more bulge surface brightness distributions are needed for comparison with those of ellipticals. Both the direct contribution of the disk to the observed luminosity distribution and the dynamical effect of the disk's potential (see e.g. Monet, Richstone and Schechter 1981) must be considered when making such comparisons. Third, are bulges intrisically more flattened than ellipticals ? Kormendy and Illingworth (1982) suggested that they are, as have Dressler and Sandage (1983). However, Boroson's (1981) surface photometry suggests that they are less flattened. More decomposition studies are required.

There remains one outstanding difference between ellipticals and bulges. "Box" - or "peanut" - shaped bulges (e.g., NGC 128, the prototypical "peanut" bulge) are common. An example, NGC 1381, an edgeon S0 in Fornax with a "box" - shaped bulge is shown in Figure 9 (see also

Figure 9. Printed from a IIa-0 + GG13 plate of NGC 1381, an edge-on so galaxy in Fornax. The "box" -shape of the bulge is apparent in the flattening of the bulge isophotes at $Z \sim 10^{\prime \prime}$ above the plane. I am grateful to K. Freeman for this print.

Davies and Illingworth 1983b). However, ellipticals with such a characteristic shape are unknown (to me at least!), while a significant fraction of bulges are "box" -shaped (10-20\%? - it would be valuable to quantify this). This structure is unlikely to be due to any direct dyna- 
mical effect of the disk upon the bulge. Such "box" -shaped structure occurs at small radii where the potential would appear to be dominated by the bulge. It has been suggested that the "box" -shaped bulge of NGC 4565 is a bar seen end-on (Jensen and Thuan 1983). Simple morphological arguments regarding the frequency of occurence of bars, their size, and the lack of thick (in z) components of that size in edge-on galaxies indicate that bars, and their associated lenses, are a stellar component that has no appreciable $z$ thickness, i.e., they are "cold" in $z$, like disks and are therefore highly triaxial (see, e.g., Kormendy 1982).

Another feature of "box" -shaped bulges that distinguishes them from other bulges and ellipticals is that there appears to be a $1: 1$ correspondence between the existence of "box" - or "peanut" -shaped structure and differential cylindrical rotation, i.e., rotation that is constant, or nearly so, with height $z$ above the plane (Kormendy and Illingworth 1982). Again, the sample is smal1, namely 3 galaxies but indicative (see Davies and Illingworth 1983a for more discussion). "Normal" bulges and ellipticals show $\mathrm{V}(\mathrm{z})$ profiles that decreases with $z$ (Illingworth and Schechter 1982, Kormendy and Illingworth 1982, Davies and Illingworth 1983c). Observational and theoretical work on these "box" -shaped bulges could be very rewarding.

While ellipticals and bulges are similar dynamically, it appears that significant differences in detail do exist.

Much of this discussion is qualitative. This is of necessity in many areas, since little data exist, and our theoretical understanding is even less. However, we have learned much in the last few years. Hopefully we will consolidate these observational gains and improve our understanding of the structure of these galaxies, and the proceses by which they formed.

\section{ACKNOWLEDGMENTS}

I am grateful to numerous colleagues for stimulating discussions. In particular, I would like to thank J. Binney, R. Davies, A. Dressler, G. Efstathiou, S. Faber, M. Fall, K. Freeman, G. Lake, C. Norman,

D. Richstone and P. Schechter.

\section{REFERENCES}

Bertola, F. : 1981, in S.M. Fall and D. Lynden-Bel1 (eds.), The Structure and Evolution of Normal Galaxies, Cambridge University Press, Cambridge, p. 13.

Bertola, F.: 1983, this volume.

Bertola, F. and Galletta, G. : 1978, Astrophys. J. Letters, 226, L115. Binney, J. : 1978, Monthly Notices Roy. Astron. Soc., 183, 779. Boroson, T.: 1981, Astrophys. J. Supp1., 46, 177. 
Davies, R.L., Efstathiou, G., Fal1, S.M., Illingworth, G. and Schechter, P.L. : 1983, Astrophys. J., 266, p. 000.

Davies, R.L. and Illingworth, G. : 1983a, Astrophys. J., 266, p.000.

Davies, R.L. and Illingworth, G. : 1983b, Astron. J., in preparation.

Davies, R.L. and I1lingworth, G. : 1983c, Astrophys. J., in preparation.

Dressler, A. : 1979, Astrophys. J., 231, 659.

Dressler, A. and Sandage, A. : 1983, Astrophys. J., in press.

Efstathiou, G. : 1982 , private communication.

Fried, J. and Illingworth, G. : 1983a, Astrophys. J. Letters, in preparation.

Fried, J. and Illingworth, G. : 1983b, Astrophys. J., in preparation.

Illingworth, G. : 1981 , in S.M. Fall and D. Lynden-Bell (eds.), The Structure and Evolution of Normal Galaxies, Cambridge University Press, Cambridge, p. 27.

Illingworth, G. and Davies, R.L. : 1983, Astrophys. J. Letters, in preparation.

I11ingworth, G. and Schechter, P.L. : 1982, Astrophys. J., 256, 481.

Jedrzejewski, R., Davies, R.L. and Illingworth, G. : 1983, Monthly Notices Roy. Astron. Soc., in preparation.

Jensen, E.B. and Thuan, T.X. : 1983, Astrophys. J., in press.

Kormendy, J. : 1982, in L. Martinet and M. Major (eds.), Morphology and Dynamics of Galaxies, Twelth in the Saas-Fee Lecture Series, Geneva Observatory, in press.

Kormendy, J. and Illingworth, G. : 1982, Astrophys. J., 256, 460.

Lake, G. : 1979, in D.S. Evans (ed.), Photometry, Kinematics and Dynamics of Galaxies, Department of Astronomy, University of Texas at Austin, p. 381 .

McEIroy, D.B. : 1983, Astrophys. J., in press.

Monet, D.G., Richstone, D.0. and Schechter, P.L. : 1981, Astrophys. J. $245,454$.

Marchant, A.B. and 01son, D.W. : 1979, Astrophys. J. Letters, 230, L157.

Merritt, D. : 1982, Astron. J., 87, 1279.

Richstone, D.0.: 1979, Astrophys. J., 234, 825.

Schechter, P.L. and Gunn, J.E. : 1978, Astron. J., 83, 1360.

Schechter, P.L. and Gunn, J.E. : 1979, Astrophys. J., 229, 472.

Steiman-Cameron, T.Y. and Durisen, R.H. : 1983, Astrophys. J. Letters, in press.

Terlevich, R., Davies, R.L., Faber, S.M. and Burstein, D. : 1981 , Monthly Notices Roy. Astron. Soc., 196, 381.

Tohline, J.E., Simonson, G.F. and Caldwe11, N. : 1982, Astrophys. J., 252,92 .

Tonry, J.L. : 1983, Astrophys. J., 264, p. 000.

Tonry, J.L. and Davis, M. : 1980, Astron. J., 84, 1511.

U1rich, M.H. and Schechter, P.L. : 1983, Astrophys. J., in preparation.

Whitmore, B.C. : 1980, Astrophys. J., 242, 53.

Williams, T.B. : 1981, Astrophys. J., 244, 458. 
THUAN : What is the light distribution along the minor axis in the, "bulge" component of the box-shaped galaxies ? Do they follow the $\mathrm{r}^{24}$ law like "normal" spheroids do ? I know that at least in the case of NGC 4565, the light drop-off along the minor axis is exponential, which is much steeper than the $\mathrm{r}^{1 / 4}$ law and which suggests that the "bulge" component in NGC 4565 is not a "normal" spheroid.

ILLINGWORTH : The evidence for the light distribution in spheroids being well-fit by an $\mathrm{r}^{1 / 4}$ law is minimal. More data are needed. The light distributions of the "box-shaped" bulges do not appear to differ in a consistent sense from the $\mathrm{r}^{1 / 4}$ law. In the outer parts, the light distributions in NGC 4565 and NGC 4111 differ in opposite senses from an $r^{1 / 4} 1 \mathrm{aw}$, one showing a deficit, the other an excess. The "box-shaped" spheroids are clearly not "normal". However, they are very common, and we need to understand why such shaped objects are only seen in disk galaxies. Why are there no "box-shaped" elliptical galaxies?

INAGAKI : In your diagrams some galaxies have velocity dispersions decreasing inwards. Do you think that these observations are reliable ? It seems to me that it is difficult to construct a dynamical model which has such a velocity dispersion distribution profile.

ILLINGWORTH : I do not think that there is any statistically significant example of a velocity dispersion profile that decreases inwards near the center of a galaxy. A constant M/L $\mathrm{r}^{1 / 4}-1$ aw galaxy should show such a decrease for $r \leqslant r_{e} / 20$ ( $1 "-3^{\prime \prime}$ for typical ellipticals). However, this type of effect would be very difficult to observe because seeing becomes a problem at such small radii. Changes on larger scales in the sense described would imply either that the $M / L$ is increasing outwards or that the azimuthal component of the velocity dispersion is preferentially increasing at the expense of the radial component.

SELLWOOD : What type of stellar population are you measuring in the disk of NGC 488 ?

ILLINGWORTH : This is very difficult to say because the data have quite low signal-to-noise. Disks are low surface brightness objects! The hydrogen 1 ines do not appear to be strong, yet the metal lines appear to be weaker than in the bulges and ellipticals that we have studied. Our solutions did however give quite acceptable values for the line strength parameter.

RICHSTONE : Is there any believable evidence for rotation along the minor axis or for velocity dispersion rising with height above the major axis?

ILLINGWORTH : The best example is NGC 596, studied extensively by Ted Williams, (published in Astrophys. J. 1981, 244, 458). Others exist but 
usually they are ellipticals that appear to be disturbed or have some photometrically striking features. "Normal" ellipticals do not show minor axis rotation, in general. However, the sample is small. The few cases that have been studied show that the velocity dispersion is either constant or decreases with distance from the major axis.

SIMKIN : Although you have not included them in your discussion, I would like to note that the $c D$ radio galaxies for which $I$ have measured absorption line velocities show very large rotations comparable to that found for the disks of Sa galaxies (peak V sini velocities of $150-350 \mathrm{~km} / \mathrm{s}$ ).

ILLINGWORTH : These objects are quite an enigma. Such large values of $\mathrm{V} / \sigma$ are more consistent with disks than spheroidal systems. If true, they would suggest that the highest radio luminosity $\mathrm{cD}$ galaxies are So's. Further data on these objects would be valuable. 